\title{
AVAILABILITY AND RELIABILITY ANALYSIS OF PROTON IMPROVEMENT PLAN-II SUPERCONDUCTING RADIO FREQUENCY LINEAR ACCELERATOR *
}

\author{
R. Prakash ${ }^{\dagger \#}$, A. Saini, G. Wu, Fermilab, Batavia, IL 60510, U.S.A. \\ ${ }^{\#}$ Also at RRCAT, Indore, MP, India and HBNI, Mumbai.
}

\section{Abstract}

Proton Improvement Plan -II is high intensity and high energy proton accelerator which will be used to support neutrino program at Fermilab. Due to the requirement from experiments, this accelerator is designed to be a highly reliable and available system. In this paper, a reliability model has been created which depicts a complete accelerator system and supporting utilities. Reliability and Availability of the complete system are estimated. It has been shown that Availability and Reliability of the system can be increased significantly by introducing failure tolerance of the beamline elements in each accelerating section of superconducting linac.

\section{INTRODUCTION}

The Proton Improvement Plan (PIP) -II is a high intensity proton facility being developed to support a neutrino program over the next two decades at Fermilab. At its core is the design and construction of a Continuous Wave $(\mathrm{CW})$ compatible superconducting radio frequency (SRF) linear accelerator (Linac) that would accelerate an average beam current of $2 \mathrm{~mA}$ up to $800 \mathrm{MeV}$. A schematic of the Linac's architecture is shown in Fig. 1. It is composed of a warm front-end and an SRF accelerating section. The warm front-end consists of an ion source (IS), a Low Energy Beam Transport (LEBT) line, an RFQ and, a Medium Energy Beam Transport line (MEBT). Most of the beam manipulations happen in this part of the Linac.



Figure 1: Acceleration scheme in the PIP-II linac. Redcoloured sections operate at room temperature while bluecoloured sections operate at $2 \mathrm{~K}$.

The beam acceleration occurs mainly in the SRF Linac that utilizes five families of superconducting cavities to accelerate the $\mathrm{H}$ - ion beam from a kinetic energy of 2.1 $\mathrm{MeV}$ to $800 \mathrm{MeV}$. Based on these families, the SRF Linac is segmented into five sections i.e. Half Wave Resonator (HWR), Single Spoke Resonator (SSR) 1 \& 2, Low Beta

\footnotetext{
* This document was prepared by [PIP-II Collaboration] using the resources of the Fermi National Accelerator Laboratory (Fermilab), a U.S. Department of Energy, Office of Science, HEP User Facility. Fermilab is managed by Fermi Research Alliance, LLC (FRA), acting under Contract No. DE-AC02-07CH11359.

†rprakash@fnal.gov
}

(LB) and High Beta (HB). The number of cryomodules (CM) and their configurations in each section are summarized in Table 1. Note that superconducting solenoids are used in the HWR, SSR1 and SSR2 sections while warm quadrupole doublets are utilized in the LB and HB sections. A detailed description of the PIP-II Linac was presented elsewhere [1].

Table 1: Numbers of elements and energy range in each section of the PIP-II SRF linac.

\begin{tabular}{lccl}
\hline Section & CM & $\begin{array}{c}\text { Cav/Mag per } \\
\text { CM }\end{array}$ & Energy (MeV) \\
\hline HWR & 1 & $8 / 8$ & $2.1-10$ \\
SSR1 & 2 & $8 / 4$ & $10-32$ \\
SSR2 & 7 & $5 / 3$ & $32-177$ \\
LB & 9 & $4 / 1^{*}$ & $177-516$ \\
HB & 4 & $6 / 1^{*}$ & $516-833$ \\
\hline one warm quadrupole doublet & located between \\
cryomodules in LB and HB sections
\end{tabular}

Success of an accelerator facility is usually measured in terms of the beam availability. A high beam reliability is essential to support ongoing experiments and to reduce unscheduled down times. Many element and utilities which are essential to accelerator operation, contribute towards the likelihood of an operation failure that may lead to an impromptu interruption. This in turn, requires consideration of these failures in the design, construction and, operation to achieve a targeted availability of the facility. In this paper, we present the availability and reliability analyses that enable us to make a quantitative assessment of the mean time to failure, availability and reliability of the PIP-II accelerator facility. Furthermore, this study also proposes way to increase the availability and reliability of the linac by introducing cavity failure tolerances. The availability goals for the PIP-II system is $90 \%$.

\section{Key Definitions}

Let us assume that $X(t)$ is state variable associated with a component which has two states of operation Working or Failed. Here, $t$ is the point of time at which state of operation is defined. Figure 2 shows the graph for $X$ as a function of time. In the Fig. 2, Time to Failure $(T)$, Time between failure $(B)$ and Time to Repair $(R)$ are shown. The statistical average value of $\langle T\rangle,\langle B\rangle$ and $<R>$ is known as Mean Time to Failure (MTTF), Mean Time Between Failure (MTBF) and Mean Time to Repair (MTTR) [2]. It should be noted that the MTTF is different 
from the lifetime. For any component MTTF represents its reliability against the failures. It is possible to have a component with large MTTF number but short lifetime.



Figure 2: Plot of State function vs time.

For a large sample size, $T$ follows a probability distribution which is known as failure probability density (f). For reliability and availability calculations, we need to select a suitable analytical expression for the probability density function. In this paper, to keep the analysis simple, $f$ was assumed to be an exponential function which is a default choice for the case when failure rate function $(\lambda)$ is a constant with time. Further, reliability or survivor function $(\mathrm{R})$, which is the probability that a system works with defined parameters for a specified period without any failure, can be defined as $e^{-\lambda t}$. Similarly, Availability is defined as the probability that the system is in working condition at any point of time or for a time period. If only corrective maintenance is done on a system, availability of the system is classified as inherent availability and it can be calculated using formula $\langle T\rangle /(<T\rangle+<B>)[3]$.

\section{RELIABILITY MODEL FOR PIP-II}

An extensive model of the PIP-II Linac facility was developed that includes not only accelerator components but also utilities that is comprised primarily of the electric-power source and its distribution, water distribution, cryogenic plant and its distribution. Figure 3 shows a simplistic top-level block-diagram representation of the model. Each major configuration unit/section is represented in the form of a block that includes components and utilities required for nominal operation of the respective unit. Figure 4 shows a detailed view of the HWR section that includes eight SRF cavities and eight superconducting solenoids. Each solenoid magnet and cavity package were further divided into their supporting components. The model is a simplistic representation of the PIP-II linac which incorporates a few assumptions and simplifications which are described as following,

\section{Assumptions and Simplifications}

1. Each block in the model represents a component and it can have binary states of operation - Working or Failed. As discussed in previous section, components have a constant failure rate during their operation hence no degraded performance is considered.

2. For analytical results of Markov chain to be applicable, failure probability of any component at any time doesn't depend on its history of operation.
3. Corrective maintenance is done when a component is failed. After repairing process, the component is restored to as good as new condition.

4. MTTR doesn't include the time spent on fault detection and lead time to get spares.

5. Reliability and availability are calculated for steady state operation of the linac. Only those failures have been considered which stop the accelerator from operation for long time. When such an operational failure occurs, a maintenance process is launched. Temporary stops of operation e.g. quenching of superconducting cavities are not included in the model. No maintenance is launched in such cases.

6. Scheduled maintenance, common cause failures and real state infrastructure has not been included in the model.



Figure 3: Top level block diagram for PIP-II Linac.

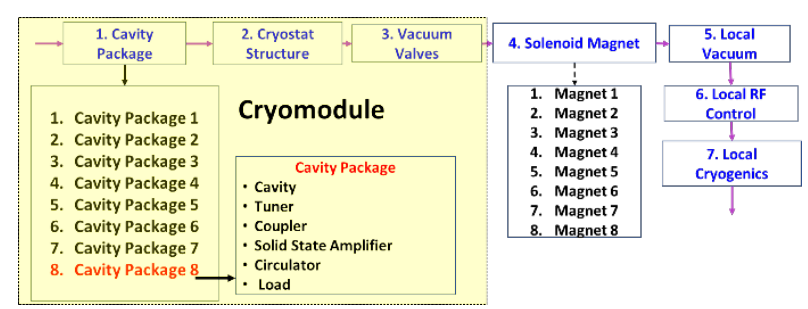

Figure 4: A detailed view of the HWR section in the PIPII Linac.

\section{Component Selection}

The accelerator has large number of components which are inter dependent in a complex manner. To simplify the model, only components exhibiting the following characteristics were included in the analysis.

1. Components used in pulsed operation,

2. Components which are thermally cycled,

3. Components which have moving parts,

4. Components which have a high stored energy,

5. Components which switches large currents (e.g. modulators),

6. Commercial parts since commercial parts may not be designed for good reliability,

Note that, these components are more vulnerable to a failure and drive the overall availability of the machine. MTTF and MTTR data for a few components in the system are commercially available and can be readily obtained. But for others, educated guesses and operational experience of subject matter experts is used. 


\section{RESULTS}

In this study, availability of the system is evaluated during steady state operation. The maintenance plan consists of only corrective maintenance activities and therefore, the analyses results consist of the inherent availability of the system. An Excel sheet method was adopted to evaluate the inherent availability of the PIP-II Linac. Because MS Excel is prone to human errors, an equivalent python-based calculation routine was developed for availability calculation. It is found that the final availability of the PIP-II Linac facility is $88.5 \%$ which is increased to $92.1 \%$ after including a failtolerance of the SRF cavities in the model. Here, failure tolerance of one cavity in each section of the superconducting part of the linac was assumed. Figure 5 shows availability break-out of the major sections and components. Table 2 summarizes the MTTF and availability of the number for the parts of the linac.



Figure 5: Availability of each configuration section of the Linac for the two cases i.e. with (blue) and without failtolerance (Red).

Table 2: Availability and MTTF value for warm section and superconducting (SC) section of the linac calculated using Excel and verified with python program.

\begin{tabular}{|c|c|c|c|c|}
\hline \multirow[b]{2}{*}{$\begin{array}{l}\text { Part of the } \\
\text { Linac }\end{array}$} & \multicolumn{2}{|c|}{ No Fail Tol } & \multicolumn{2}{|c|}{ Incl. Fail Tol. } \\
\hline & $\begin{array}{c}\text { MTTF } \\
\text { (h) }\end{array}$ & $\begin{array}{c}\text { Avail. } \\
(\%)\end{array}$ & $\begin{array}{l}\text { MTTF } \\
\text { (h) }\end{array}$ & $\begin{array}{l}\text { Avail. } \\
(\%)\end{array}$ \\
\hline Utility & 1741.55 & 97.40 & 1741.55 & 97.40 \\
\hline Warm Section & 127.82 & 95.73 & 127.82 & 95.73 \\
\hline SC Section & 126.75 & 94.73 & 188.46 & 98.82 \\
\hline Total Linac & 61.40 & 88.33 & 72.97 & 92.14 \\
\hline
\end{tabular}

\section{Basic Reliability}

Reliability of a system indicates the robustness of the system against possible failures. In this section, the reliability of the PIP-II Linac is calculated. Figure 6 shows the reliability of the complete system as a function of time. The two curves in the figure consist of two different configurations of the model, i.e. with and without fail-tolerance of SRF cavities. As one can expect, the reliability increases after inclusion of the failtolerances of the SRF cavities. As the Figure shows, the model predicts the PIP-II Linac reliability of $22.8 \%$ over the period of $100 \mathrm{~h}$. Reliability data is summarized in the Table 3.

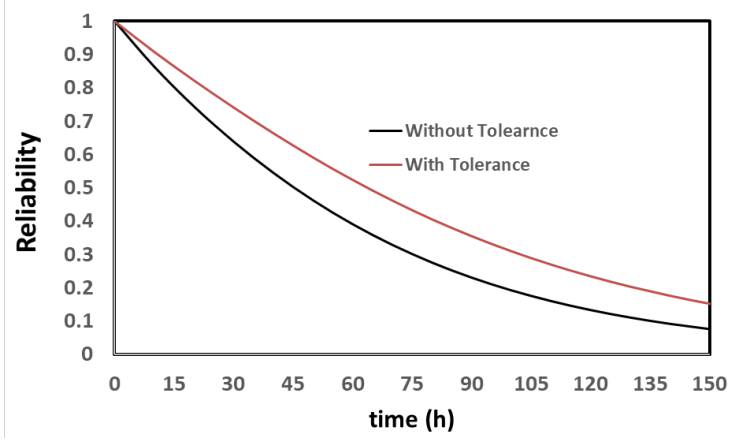

Figure 6: Reliability of the PIP-II Linac with (black) and without (red) fail-tolerance of SRF cavity.

Table 3: Reliability of linac parts at $100 \mathrm{~h}$.

\begin{tabular}{ccc}
\hline \multirow{2}{*}{ Part of Linac } & \multicolumn{2}{c}{ Reliability (\%) } \\
\cline { 2 - 3 } & No Fail Tol. & With Fail Tol \\
\hline Warm Section & 53.20 & 53.20 \\
SC Section & 45.43 & 73.26 \\
Total Linac & 22.82 & 36.80 \\
\hline
\end{tabular}

\section{CONCLUSIONS}

Availability and reliability analysis are an important part of the accelerators which have high availability in the system design. The target availability of the PIP-II linac is $90 \%$. A model has been created to estimate the reliability and availability numbers for PIP-II. The model is based on a tree kind of structure where the complete system is divided into a several section and each section is further divided into substructure, until the layer where data exists is reached. Calculations for this model were performed using Excel sheet and verified with python-based code. We obtain a $22.8 \%$ reliability at $100 \mathrm{~h}$ and $88.3 \%$ availability for the PIP-II linac. Failure tolerance of one cavity per section was introduced which enhanced the availability to $\sim 92.1 \%$ and reliability to $\sim 36.8 \%$.

\section{ACKNOWLEDGEMENTS}

Authors takes this opportunity to acknowledge S. Luigi, CERN for the critical reviews of the work. Author also thank to A. Klebaner, A. Martinez and entire PIP-II team for useful suggestions and discussions.

\section{REFERENCES}

[1] The DUNE Collaboration, "CDR Volume 1: The LBNF and DUNE Projects," tech. rep., 2015.

[2] M. Rausand and A. Hoyland, "System Reliability Theory, Hobken, New Jersey: John Wiley \& Sons, 2004.

[3] S. Bhattacharya, "Reliability Analysis and Controls for Accelerator Driven Systems based on Project X," Ohio State Univeristy, 2012. 